\title{
New Lower Bounds for Orthogonal Graph Drawings *
}

\author{
Therese C. Biedl \\ RUTCOR, Rutgers University, P.O. Box 5062, New Brunswick, NJ 08903-5062, \\ therese@rutcor.rutgers.edu
}

\begin{abstract}
An orthogonal drawing is an embedding of a graph such that edges are drawn as sequences of horizontal and vertical segments. In this paper we explore lower bounds. We find lower bounds on the number of bends when crossings are allowed, and lower bounds on both the grid-size and the number of bends for planar and plane drawings.
\end{abstract}

\section{Introduction}

Orthogonal graph drawings are an important tool for graph layout, e.g. for Data Flow Diagrams or Entity Relationships Diagrams. Two important measurements of the quality of a drawing are the grid-size and the number of bends. Every 4graph has an orthogonal drawing of grid-size $\mathcal{O}(n) \times \mathcal{O}(n)$ with $\mathcal{O}(n)$ bends. Minimizing the number of bends is $\mathcal{N P}$-complete [6], and so is the question whether a graph can be embedded in a grid of prescribed size $[8,5]$. Therefore, one tries to find heuristics where the obtained worst-case sizes are a priori known and small. Different algorithms have been developed, depending on the connectivity and whether the graph is planar or not. See Table 1 for an overview.

\begin{tabular}{|c|c|c|c|c|c|c|}
\hline & \multicolumn{2}{|c|}{ Triconnected } & \multicolumn{2}{|c|}{ Biconnected } & \multicolumn{2}{|c|}{ Connected } \\
\hline & Grid-size & Bends & Grid-size & Bends & Grid-size & Bends \\
\hline & \multicolumn{6}{|c|}{ Nonplanar } \\
\hline Simple & $\operatorname{lp} \frac{7}{4} n-2^{*}$ & $2 n+2[1]$ & hp $\frac{7}{4} n-2^{*}$ & $2 n+2[1]$ & $n[1]$ & $2 n+2[1]$ \\
\hline Multigraph & $n+1[1]$ & $2 n+4[1]$ & $n+1[1]$ & $2 n+4[1]$ & $\frac{4}{3} n-1[4]$ & $\frac{8}{3} n+2[4]$ \\
\hline \multirow[t]{2}{*}{ With Loops } & \multicolumn{2}{|l|}{-} & $2 n-1[4]$ & $2 n-1[4]$ & $4 n[4]$ & $4 n[4]$ \\
\hline & \multicolumn{6}{|c|}{ Plane } \\
\hline Simple & hp $\frac{4}{3} n+2[4]$ & $\frac{4}{3} n+4[4]$ & $n[11]$ & $2 n+2[2]$ & $\frac{6}{5} n+1[4]$ & $\frac{12}{5} n+2[4]$ \\
\hline Multigraph & $n+1[13]$ & $2 n+4[14]$ & $n+1[13]$ & $2 n+4[14]$ & $2 n-1[4]$ & $4 n-2[4]$ \\
\hline With Loops & - & & $2 n+1[4]$ & $4 n+4[4]$ & $2 n+1[4]$ & $4 n+4[4]$ \\
\hline
\end{tabular}

Table 1. Known algorithms. "hp" means that this is a bound on the half-perimeter. We give the (to our knowledge) first citation of each result. The results marked * are by Papakostas and Tollis (private communication).

* A full version of this paper can be found in [3]. This paper was written while the author was visiting TU Berlin and working at Tom Sawyer Software. 
To measure the goodness of these algorithms, we want to find graphs which need at least a certain grid-size or at least a certain amount of bends. In this paper we deal with these lower bounds. We summarize our results in Table 2.

\begin{tabular}{|l|l||c|c|c|c|}
\hline \multicolumn{2}{|c|}{ Nonplanar Drawings } & Triconnected & Biconnected & Connected & Non-Connected \\
\hline \hline \multirow{2}{*}{ Non-planar } & Simple & $\frac{10}{7} n$ & $\frac{10}{6} n$ & $\frac{11}{6} n$ & $\frac{12}{5} n$ \\
\hline \hline \multirow{3}{*}{ Planar } & Simple & $\frac{6}{5} n$ & $\frac{10}{7} n$ & $\frac{11}{7} n$ & $2 n$ \\
\cline { 2 - 6 } & Multigraph & $\frac{10}{7} n$ & $2 n$ & $\frac{7}{3} n$ & $4 n$ \\
\cline { 2 - 6 } & With Loops & - & $3 n$ & $3 n$ & $6 n$ \\
\hline
\end{tabular}

\begin{tabular}{|c|l||c|c|c|c|}
\hline \multicolumn{2}{|c|}{ Plane Drawings } & Triconnected & Biconnected & Connected & Non-Connected \\
\hline \multirow{3}{*}{ Simple } & Grid-size & $\frac{2}{3} n+1$ & $n-1$ & $\frac{6}{5}(n-1)-1$ & $?$ \\
\cline { 2 - 6 } & Bends & $\frac{4}{3} n+4$ & $2 n-2 *$ & $\frac{12}{5}(n-1)-2$ & $?$ \\
\hline \hline \multirow{3}{*}{ Multigraph } & Grid-size & $\frac{2}{3}(n-2)+3$ & $n+1$ & $2 n-3$ & $2 n-1$ \\
\cline { 2 - 6 } & Bends & $\frac{4}{3}(n-2)+8$ & $2 n+4 *$ & $4 n-6$ & $4 n$ \\
\hline \multirow{2}{*}{ With Loops } & Grid-size & - & $n+2$ & $2 n+1$ & $4 n-1$ \\
\cline { 2 - 6 } & Bends & - & $3 n$ & $4 n+4$ & $6 n$ \\
\hline
\end{tabular}

Table 2. Lower bounds for orthogonal drawings. "-" means that this case is impossible. "?" means that we didn't find lower bounds better than for the connected case. The results marked $*$ were already discovered by [15].

\section{Definitions}

Let $G$ be a graph with $n$ vertices. We always assume that $G$ is a 4 -graph, i.e. it has a maximum degree of $4 . G$ is called 4-regular if every vertex has degree 4 . By subdivision of an edge $e$ we understand that we delete $e$, add a new vertex, and connect it with the two endpoints of $e$. Edges of the form $(v, v)($ loops) are not necessarily forbidden. Also, two vertices may be connected by more than one edge (multiple edge). Graphs without loops and multiple edges are called simple, graphs without loops, but possibly with multiple edges, are called multigraphs.

$G$ is called connected if for any two vertices there is a path between them. It is called biconnected if for any vertex $v$ the graph $G-\{v\}$ is connected. It is called triconnected if for any two vertices $v, w$ the graph $G-\{v, w\}$ is connected. A triconnected 4-graph with more than three vertices can never have a loop.

A graph is called planar if it has a drawing without crossing (planar drawing). This defines a circular ordering of the edges incident to a vertex $v$ (combinatorial embedding). A planar drawing splits the plane into different components, called faces. The unbounded component is called the outerface. The combinatorial embedding defines a planar drawing which is unique except for the choice of the outerface. A planar graph is called plane if both a combinatorial embedding and the outerface are specified. 
An (orthogonal) drawing of $G$ is an embedding of $G$ in the plane such that all edges are drawn as sequences of horizontal and vertical line segments. It is called planar if no drawings of edges intersect. It is called plane if it is planar, if $G$ was a plane graph, and the drawing exactly reflects the given embedding and the outerface. A point where the drawing of an edge changes its direction is called a bend of this edge.

A column (row) of the drawing is called vertex-used if it contains a vertex, line-used if it contains a vertical (horizontal) part of an edge, and used if it is vertex-used or line-used. The width of the drawing is the number of used columns minus 1 , the height is the number of used rows minus 1 . A drawing with width $n_{1}$ and height $n_{2}$ has grid-size $n_{1} \times n_{2}$, half-perimeter $n_{1}+n_{2}$, and area $n_{1} \cdot n_{2}$.

\section{Lower Bounds for Non-Planar Drawings}

Very little is known about lower bounds for the grid-size. There exist 4-graphs with crossing number $\Omega\left(n^{2}\right)$ which therefore need a grid of the same area [16]. Leighton [9] proved that the planar tree of meshes needs $\Omega(n \log n)$ area in any orthogonal drawing. In both cases the constants are very small.

We deal here with the number of bends, and develop various lower bounds, depending on the connectivity of the graph. The only known results are for simple biconnected graphs: Storer [11] showed a lower bound of $\frac{8}{7} n$ bends, and Papakostas and Tollis improved it to $\frac{8}{5} n$ (private communication).

\subsection{Lower Bounds for Small Graphs}

We use the following special graphs: The complete graph $K_{5}$, the octahedron $O$, the quadruple edge graph $Q$, and the double loop $L$, which are shown below. We develop a few easy lemmas to get lower bounds for these graphs.
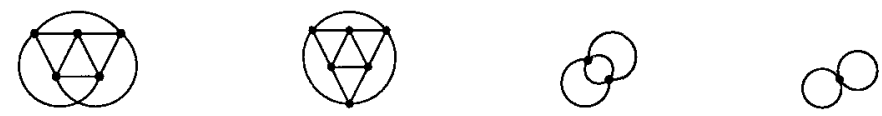

Fig. 1. $K_{5}$, the octahedron, the quadruple edge, and the double-loop.

Lemma 1. In a drawing of a 4-regular graph, every used column has two bends. Formulated differently: if a drawing of a 4-regular graph has width $w$, then there are at least $2 w+2$ bends.

Proof. If a column is vertex-used, let $u$ be the top vertex and $w$ be the bottom vertex in the column. By 4-regularity all connections at $u$ and $w$ are used. So the top connection from $u$ must have a bend, and so must the bottom connection of $w$. If a column is line-used only, every begin- and endpoint of a line is a bend. Hence we have at least two bends.

In a drawing of width $w$ there are $w+1$ used columns (by definition of width), and therefore at least $2 w+2$ bends by the above. 
Lemma 2. A 4-regular graph has $2\lceil\sqrt{n}\rceil+4$ bends in any drawing.

Proof. We only sketch this proof. It is clear that we need at least either $\lceil\sqrt{n}\rceil$ columns or $\lceil\sqrt{n}\rceil$ rows to accomodate the vertices. Since we have a 4-regular graph, we need two more rows and two more columns at the extreme ends. This proves the claim together with Lemma 1.

Lemma 3. A simple 4-regular graph has at least 12 bends in any drawing.

Proof. The proof is an easy (but lengthy) case analysis to show that in any embedding we must have at least 6 rows or 6 columns. We then have at least 12 bends by Lemma 1 (or analogously for rows). We skip this for brevity.

These two lemmas imply that $L$ needs 6 bends, $Q$ needs 8 bends, and $K_{5}$ and $O$ need 12 bends in any drawing.

\subsection{Constructing bigger graphs}

The lower bounds for non-connected graphs are easy to get by taking many copies of $O, K_{5}, Q$, and $L$, respectively. To obtain bigger connected graphs, we need to study how subdividing an edge changes the lower bound.

Lemma 4. Subdividing an edge lowers the lower bound on the bends by at most 1 .

Proof. Assume $G$ needs $b$ bends, and after subdividing one edge, we get $G_{s}$. Let $G_{s}$ be drawn with $c$ bends. If we remove the vertex that came from subdividing, this adds at most one bend, so we get a drawing of $G$ with $c+1$ bends. Consequently $c \geq b-1$.

Theorem 5. There are the following lower bounds for connected graphs:

1. planar simple graphs: $\frac{11}{7} n$ bends

2. simple graphs: $\frac{11}{6} n$ bends

3. planar multigraphs: $\frac{7}{3} n$ bends

4. planar graphs with loops: $3 n$ bends

Proof. We demonstrate only case (1) in detail, the other cases are analogous. Take an octahedron and subdivide one edge. We get a graph with 7 vertices that by Lemma 4 needs 11 bends. Take $k$ copies and connect the vertices of degree 2 . This graph is connected, has $7 k$ vertices and needs $11 k=\frac{11}{7} n$ bends.
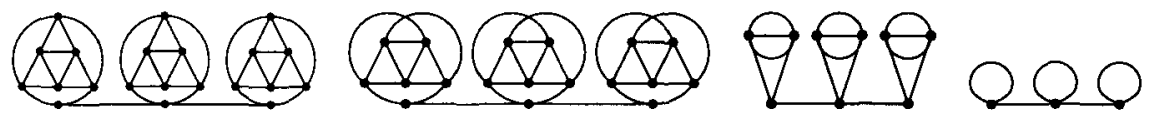

Fig. 2. Connected graphs that need many bends in any drawing. 
Theorem 6. There are the following lower bounds for biconnected graphs:

1. planar simple graphs: $\frac{10}{7} n$ bends

2. simple graphs: $\frac{10}{6} n$ bends

3. planar multigraphs: $\frac{6}{3} n=2 n$ bends

4. planar graphs with loops: $3 n$ bends

Proof. Again we demonstrate only case (1). Take an octahedron and subdivide two edges on the outerface. We get a graph with 8 vertices that by Lemma 4 needs 10 bends. Take $k$ copies and identify the vertices of degree 2 . This graph is biconnected, has $7 k$ vertices and needs $10 k=\frac{10}{7} n$ bends.
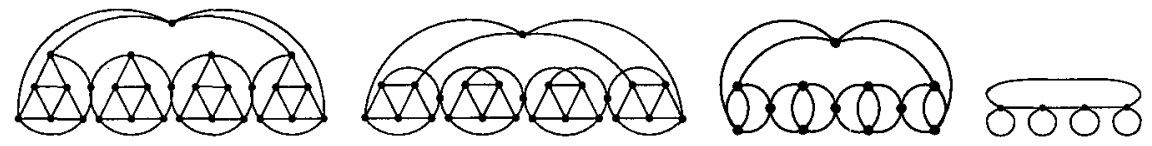

Fig. 3. Biconnected graphs that need many bends in any drawing.

Theorem 7. There are the following lower bounds triconnected graphs:

1. planar simple graphs: $\frac{6}{5} n$ bends.

2. simple graphs: $\frac{18}{13} n$ bends.

3. planar multigraphs: $\frac{18}{13}$ bends.

Proof. Again we demonstrate only case (1). Take an octahedron and subdivide the three edges on the outerface. We get a graph with 9 vertices that by Lemma 4 needs 9 bends. Take $2 k$ copies and identify the vertices of degree 2 in such a way that the resulting graph is planar and triconnected (see also Fig. 4). The graph then has $\left(6+\frac{3}{2}\right) 2 k=15 k$ vertices and needs $9 \cdot 2 k=\frac{18}{15} n=\frac{6}{5} n$ bends.
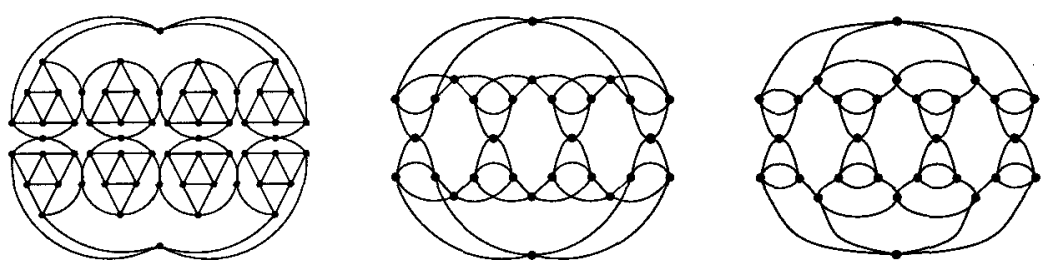

Fig. 4. Triconnected graphs that need many bends in any drawing. 


\section{Lower Bounds for Planar Drawings}

\subsection{Plane graphs}

Assume after taking away all edges on the outerface of a plane graph $H$ we get the graph $G$. Then we say that $H$ contains $G$ on the inside.

Lemma 8. Let $H$ contain $G$ on the inside, where $G$ has minimum degree 2. If $H$ can be drawn in a $w \times h$-grid then $G$ can be drawn in a $(w-2) \times(h-2)$-grid.

Proof. Consider a drawing $\Gamma_{H}$ of $H$ in a $w \times h$-grid. Deleting the edges on the outerface of $H$ we get a drawing $\Gamma_{G}$ of $G$. Since $G$ has minimum degree 2 , the highest row of $\Gamma_{G}$ must be used by a horizontal line. This line belongs to an edge $e$ on the outerface of $G$. Since $G$ is on the inside of $H, e$ is not on the outerface of $H . \Gamma_{H}$ reflects the embedding of $H$, so we must have a line drawn above $e$ in $\Gamma_{H}$. Therefore $\Gamma_{H}$ has at least one more unit in top-direction. The same holds for the other three directions.

Triconnected graphs Kant showed lower bounds of $\frac{2}{3}(n-2)+2$ on the gridsize and $\frac{4}{3}(n-2)+2$ bends for triconnected plane graphs. We improve this slightly with the following graph class:

Definition 9. Define the graph classes $\left\{T_{i}^{\prime}\right\}$ and $\left\{T_{i}\right\}$ as follows:

$-T_{1}^{\prime}$ is a 3 -cycle.

- $T_{i}^{\prime}$ is obtained by taking a copy of $T_{i-1}^{\prime}$, and adding three vertices in the outerface. Then we add a 6 -cycle between the three new vertices and the three vertices of degree 2 of $T_{i-1}^{\prime}$, such that $T_{i}^{\prime}$ contains $T_{i-1}^{\prime}$ on the inside.

- $T_{i}$ is obtained by taking a copy of $T_{i}^{\prime}$ and adding a 3-cycle between the three vertices of degree 2 of $T_{i}^{\prime}$ such that $T_{i}$ contains $T_{i}^{\prime}$ on the inside.

See Fig. 5 for an illustration of this graph.

Lemma 10. $T_{i}$ needs a width and height of $2 i+1$ in any plane drawing.

Proof. We first show a lower bound for $T_{i}^{\prime}$, namely, it needs a width of $2 i-1$. This is shown by induction on $i$. Since $T_{1}^{\prime}$ is a triangle, it needs a $1 \times 1$-grid. Now consider $T_{i}^{\prime}, i \geq 2$. By construction it contains $T_{i-1}^{\prime}$ on the inside, so if we could embed $T_{i}^{\prime}$ with width less than $2 i+1$, then by Lemma 8 we could embed $T_{i-1}^{\prime}$ in a grid of width less than $2 i-1=2(i-1)+1$, a contradiction.

Now finally consider $T_{i}$. By construction it contains $T_{i}^{\prime}$ on the inside, so it needs two more units in width than $T_{i}^{\prime}$, which gives a lower bound of $2 i+1$ on the width. The proof for the height is similar.

Lemma 11. $T_{i}$ needs $4 i+4$ bends in any plane drawing.

Proof. This is trivial: $T_{i}$ is 4-regular and has a width of $2 i+1$ in any drawing. By Lemma 1 it therefore must have $2(2 i+1)+2=4 i+4$ bends in any drawing. 
Theorem 12. There are the following lower bounds for plane triconnected graphs:

- simple graphs: $\left(\frac{2}{3} n+1\right) \times\left(\frac{2}{3} n+1\right)$-grid and $\frac{4}{3} n+4$ bends.

- multigraphs: $\left(\frac{2}{3}(n-2)+3\right) \times\left(\frac{2}{3}(n-2)+3\right)$-grid and $\frac{4}{3}(n-2)+8$ bends.

Proof. We are done in the simple case, since $T_{i}$ has $n=3 i$ vertices. Obtain graph $\hat{T}_{i}$ as follows: subdivide two different edges on the outerface of $T_{i}$, and add a double edge between the two new vertices, such that the double edge encloses $T_{i}$. Some calculation shows that $\hat{T}_{i}$ has $3 i+2$ nodes, needs a width and height of $2 i+3$, and $4 i+8$ bends. This proves the claim for multigraphs.

Biconnected graphs Storer showed a lower bound of $n-2$ on the grid-size of biconnected simple graphs [11]. Tamassia, Tollis, and Vitter showed a lower bound of $2 n+4$ bends for multigraphs and $2 n-2$ bends for simple graphs [15]. We use their graph class to show a slightly better bound on the grid-size.

Definition 13. Define the graph classes $\left\{B_{i}^{\prime}\right\}$ and $\left\{B_{i}\right\}$ as follows:

$-B_{1}^{\prime}$ is a double edge.

- $B_{i}^{\prime}$ is obtained by taking a copy of $B_{i-1}^{\prime}$, adding two vertices in the outerface, and adding a 4-cycle alternating between the two new vertices and the two vertices of degree 2 of $B_{i-1}^{\prime}$, such that $B_{i}^{\prime}$ contains $B_{i-1}^{\prime}$ on the inside.

- $B_{i}$ is obtained by taking a copy of $B_{i}^{\prime}$ and adding a double edge between the two vertices of degree 2 of $B_{i}^{\prime}$ such that $B_{i}$ contains $B_{i}^{\prime}$ on the inside.

The following lemma is proved exactly as in Lemma 10 and Lemma 11. The second claim was known before [15], though proved by different means.

Lemma 14. $B_{i}$ needs a width and height of $2 i+1$ and $4 i+4$ bends.
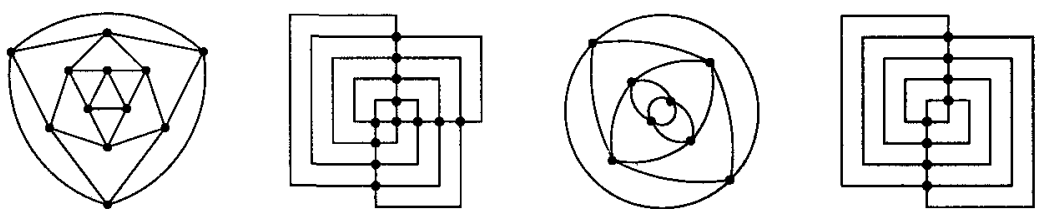

Fig. 5. $T_{4}$ and $B_{4}$, both drawn optimally.

Theorem 15. There are the following lower bounds for plane biconnected graphs:

- simple graphs: $(n-1) \times(n-1)$-grid and $2 n-2$ bends

- multigraphs: $(n+1) \times(n+1)$-grid and $2 n+4$ bends

- graphs with loops: $(n+2) \times(n+2)$-grid and $3 n$ bends 
Proof. For multigraphs we are done by Lemma 14 since $B_{i}$ has $2 i$ vertices.

For simple graphs, let $\bar{B}_{i}$ be the graph obtained from $B_{i}$ by subdividing one of each of the double edges. Some calculation shows that $\bar{B}_{i}$ has $2 i+2$ vertices, needs a height and width of $2 i+1$ and $4 i+2$ bends in any drawing.

For graphs with loops, let $\hat{B}_{i}$ be the graph obtained from $B_{i}$ by subdividing one edge on the outerface, and adding a loop incident to the new vertex, such that the loop encloses $B_{i}$. Some calculation shows that $\hat{B}_{i}$ has $2 i+1$ vertices, and needs a width and height of $2 i+3$ in any drawing. This proves the lower bound on the grid-size. For the number of bends, the claim was shown in Theorem 6.

Connected graphs The known lower bounds for connected plane graphs are $\frac{8}{3}(n-2)$ bends for multigraphs, and $4(n-2)$ bends for graphs with loops [15]. We improve these bounds and develop new ones for simple graphs.

Definition 16. Define the graph classes $\left\{C_{i}^{\prime}\right\}$ and $\left\{C_{i}\right\}$ as follows:

$-C_{1}^{\prime}$ is a loop.

- $C_{i}^{\prime}$ is obtained by taking a copy of $C_{i-1}^{\prime}$, adding one vertex in the outerface, and adding a double edge between the new vertex and the vertex of degree 2 of $C_{i-1}^{\prime}$, such that $C_{i}^{\prime}$ contains $C_{i-1}^{\prime}$ on the inside.

- $C_{i}$ is obtained by taking a copy of $C_{i}^{\prime}$ and adding a loop at the vertex of degree 2 of $C_{i}^{\prime}$ such that $C_{i}$ contains $C_{i}^{\prime}$ on the inside.

See Fig. 6 for an illustration of this graph class. The following lemma is proved exactly as in Lemma 10 and Lemma 11.

Lemma 17. $C_{i}$ needs $a$ width and height of $2 i+1$ and $4 i+4$ bends.

Definition 18. The graph class $C M_{i}$ is essentially defined as the graph class $C_{i}$, with the exception that both loops are replaced by a quadruple edge with one edge subdivided. See also Fig. 6.

The following lemma is proved similar as in Lemma 10 and Lemma 11.

Lemma 19. $C M_{i}$ needs a width and height of $2 i+5$ and $4 i+12$ bends.
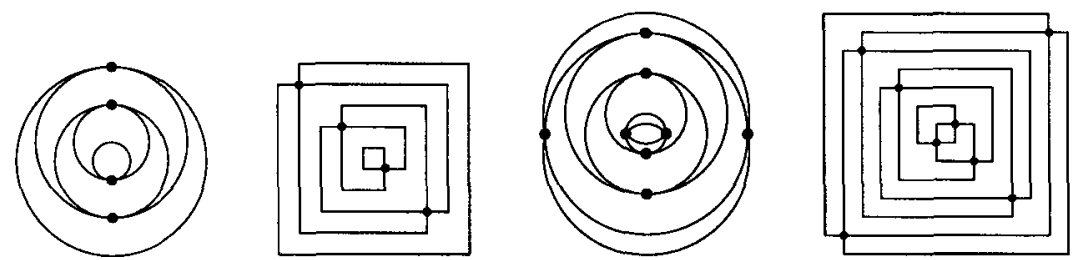

Fig. 6. $C_{4}$ and $C M_{4}$, both drawn optimally. 
Definition 20. Define the graph classes $\left\{C S_{i}\right\},\left\{C S_{i}^{\prime}\right\}$ and $\left\{C S_{i}^{\prime \prime}\right\}$ as follows:

$-C S_{1}^{\prime \prime}$ is a 3 -cycle.

- $C S_{i}^{\prime}(i \geq 1)$ is obtained by taking a copy of $C S_{i}^{\prime \prime}$ and adding two vertices in the outerface. Then we add a 4-cycle alternating between the two new vertices and two vertices of degree 2 of $C S_{i}^{\prime \prime}$, such that $C S_{i}^{\prime}$ contains $C S_{i}^{\prime \prime}$ on the inside.

- $C S_{i}(i \geq 1)$ is obtained by taking a copy of $C S_{i}^{\prime}$ and adding one vertex in the outerface. Then we add a 3-cycle between this new vertex and the two vertices of degree 2 of $C S_{i}^{\prime}$, such that $C S_{i}$ contains $C S_{i}^{\prime}$ on the inside.

- $C S_{i+1}^{\prime \prime}(i \geq 1)$ is obtained by taking a copy of $C S_{i}$ and adding two vertices in the outerface. Then we add a 3-cycle between the two new vertices and the vertex of degree 2 of $C S_{i}$, such that $C S_{i+1}^{\prime \prime}$ contains $C S_{i}$ on the inside.
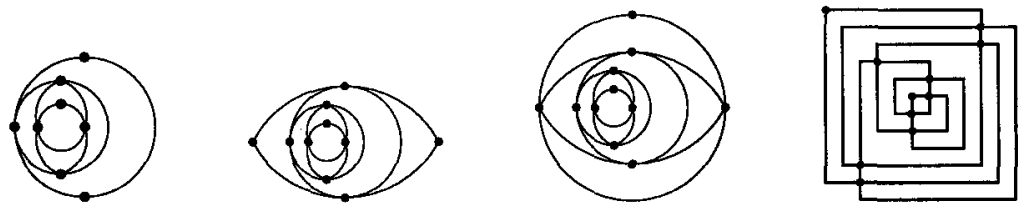

Fig. 7. $C S_{2}^{\prime \prime}, C S_{2}^{\prime}$, and $C S_{2}$; and an optimal drawing.

Lemma 21. $C S_{i}$ needs a width and height of $6 i-1$ in any plane drawing.

Proof. We show only the width by induction on $i$; showing also a lower bound for $C S_{i}^{\prime \prime}$ of $6 i-5$ and a lower bound for $C S_{i}^{\prime}$ of $6 i-3$. $C S_{1}^{\prime \prime}$ is a triangle that needs a width of $1=6 \cdot 1-5$. Assume the claim was shown for $C S_{i}^{\prime \prime}$.

$C S_{i}^{\prime}$ contains $C S_{i}^{\prime \prime}$ on the inside and needs two more units in width than $C S_{i}^{\prime \prime}$, which gives a lower bound of $6 i-3 . C S_{i}$ contains $C S_{i}^{\prime}$ on the inside and needs two more units in width than $C S_{i}^{\prime}$, which gives a lower bound of $6 i-1$. Finally, $C S_{i+1}^{\prime \prime}$ contains $C S_{i}$ on the inside and needs two more units in width than $C S_{i}$, which gives a lower bound of $6 i+1=6(i+1)-5$.

Lemma 22. $C S_{i}$ needs $12 i-2$ bends in any plane drawing.

Proof. $C S_{i}$ has two vertices of degree 2. If we remove those and connect their neighbors by an edge, we get a 4-regular graph $C S_{i}^{*}$, which needs the same width as $C S_{i}, 6 i-1$. By Lemma $1 C S_{i}^{*}$ therefore needs $12 i$ bends. $C S_{i}$ results from $C S_{i}^{*}$ by subdividing two edges, so by Lemma $4 C S_{i}$ needs $12 i-2$ bends.

Theorem 23. There are the following lower bounds for plane connected graphs:

- graphs with loops: $(2 n+1) \times(2 n+1)$-grid and $4 n+4$ bends.

- multigraphs: $(2 n-3) \times(2 n-3)$-grid and $4 n-4$ bends.

- simple graphs: $\left(\frac{6}{5}(n-1)-1\right) \times\left(\frac{6}{5}(n-1)-1\right)$-grid and $\frac{12}{5}(n-1)-2$ bends.

Proof. One shows easily that $C_{i}$ has $i$ vertices, $C M_{i}$ has $i+4$ and $C S_{i}$ has $5 i+1$ vertices. The results follow with Lemma 17, 19, 21 and 22. 
Non-connected graphs For brevity we skip the definition and proofs for nonconnected graphs. For simple graphs we did not find graphs with better lower bounds than those for connected graphs.
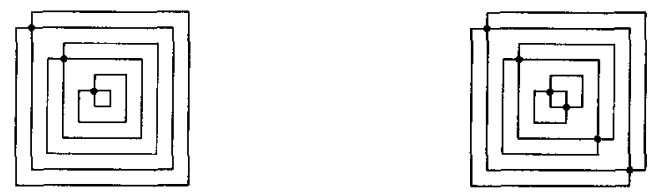

Fig. 8. Non-connected 4-graphs which need a big grid and many bends.

Theorem 24. There are the following lower bounds for plane graphs:

- graphs with loops: $(4 n-1) \times(4 n-1)$-grid and $8 n$ bends.

- multigraphs: $(2 n-1) \times(2 n-1)$-grid and $4 n$ bends.

\subsection{Combinatorial embedding can be chosen}

To the author's knowledge no research has been done into lower bounds for planar drawings. We provide some results here which are close to optimality in the number of bends.

Triconnected Graphs For triconnected planar graphs there exists only one combinatorial embedding. Therefore, if we consider all possible choices of the outerface of $T_{i}$, then we get a lower bound for any planar drawing of $T_{i}$.

Lemma 25. $T_{i}$ needs a width and height of $i$ and $4 i-2$ bends in any planar drawing.

Proof. Assume that $T_{1}^{\prime}$ had the vertices $\left\{v_{1}, v_{2}, v_{3}\right\}$ and that we obtained $T_{i}^{\prime}$ by adding the vertices $\left\{v_{3 i-2}, v_{3 i-1}, v_{3 i}\right\}$. Let $\Gamma$ be the embedding of $T_{i}$ defined in Definition 9 , this induces an embedding of $T_{i}^{\prime}$. We know that $T_{i}^{\prime}$ in this embedding needs a width of $2 i-1$, and can also show that it needs $4 i-3$ bends. Assume we are given some planar orthogonal drawing of $T_{i}$, this induces a planar embedding $\Gamma^{\prime}$. The outerface of $\Gamma^{\prime}$ can have degree 3 or 4 .

If the degree is 3 , then we may assume that the outerface of $\Gamma^{\prime}$ is either the outerface of $\Gamma$, or one of the three faces adjacent to it. After deletion of the edges $\left(v_{3 i}, v_{3 i-1}\right),\left(v_{3 i-1}, v_{3 i-2}\right),\left(v_{3 i-2}, v_{3 i}\right)$ we have a copy of $T_{i}^{\prime}$ embedded as in $\Gamma$. So we need a width and height of $2 i-1 \geq i$ and $4 i-3$ bends of $T_{i}^{\prime}$. The three deleted edges form a triangle and need a bend, so we get a lower bound of $4 i-2$ bends.

If the degree is 4 , we assume after possible renumbering that the outerface is $\left\{v_{3 j-3}, v_{3 j-1}, v_{3 j}, v_{3 j+1}\right\}$ for some $1<j<i$. Splitting the graph at $v_{3 j-2}, v_{3 j-1}$ and $v_{3 j}$ we get a copy of $T_{j}^{\prime}$ and a copy of $T_{i-j+1}^{\prime}$, embedded as in $\Gamma$. So the number of bends in this embedding is at least $4 j-3+4(i-j+1)-3=4 i-2$. At the very least the width and height of the drawing must be $\min \{2 j-1,2(i-j+1)-1\}$ which is smallest for $j=\frac{i+1}{2}$ and then equals $i$. 

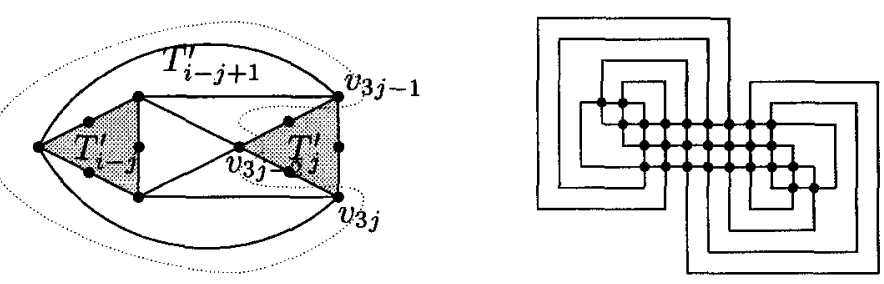

Fig. 9. $T_{i}$ embedded with outerface-degree 4 leads to a bend-optimal drawing.

Theorem 26. We have a lower bound of an $\frac{n}{3} \times \frac{n}{3}$-grid and $\frac{4}{3} n-2$ bends for planar drawings of triconnected graphs.

Biconnected graphs For biconnected graphs the combinatorial embedding is not unique. However, even though $B_{i}$ has many different combinatorial embeddings, all give the same planar drawing, except for possible renaming of the vertices and the choice of the outerface. Therefore, by considering different choices of the outerface, we get a lower bound for planar drawings of $B_{i}$.

We do not explain the details here, and leave it to the reader to show that $B_{i}$ needs an $i \times i$-grid and $4 i$ bends in any planar drawing. We can then again go over to $\bar{B}_{i}$ to get the lower bounds for simple graphs.

Theorem 27. There are the following lower bounds for planar biconnected graphs:

- multigraphs: $\frac{n}{2} \times \frac{n}{2}$ and $2 n$ bends.

- simple graphs: $\left(\frac{n}{2}-1\right) \times\left(\frac{n}{2}-1\right)$ and $2 n-6$ bends.

\section{Remarks and Open Problems}

In this paper we have considered lower bounds: for the number of bends in the non-planar case and on both the number of bends and the grid-size in the planar and plane case. Various results have been proved, which either give completely new lower bounds or considerably improved the old ones.

For plane graphs, the results are almost optimal, and the difference is only a small constant, if at all. For planar graphs, the results are fairly good in terms of the number of bends, but improvement should be possible for the grid-size.

Much work remains to be done for non-planar drawings. For the number of bends, there is a small gap in the factor between the lower and the upper bound. No algorithm is known that draws a planar graph with fewer bends if we allow for crossings. We suspect that such an algorithm should be possible.

An even bigger problem are lower bounds on the grid-size for non-planar drawings. The current proofs give only a fairly small constant. It would be also interesting to see more techniques for proving lower bounds on the grid-size of non-planar drawings.

Finally, we would like to pose the open problem of lower bounds for graphs of higher maximum degree. Usually, graphs with higher degree are drawn orthogonally by assigning boxes instead of points to vertices. With such a representation 
every planar graph can be drawn without bends (see $1 \mathrm{D}$ visibility representations $[10,12])$. But not all graphs can be drawn without bends: such a drawing is a $2 \mathrm{D}$ visibility representation, and can exist only for graphs which are the union of two planar graphs. It would be interesting to see which graphs can be drawn without bends at all, and what are lower bounds for those that can't.

\section{References}

1. T. Biedl, Embedding Nonplanar Graphs in the Rectangular Grid, Rutcor Research Report 27-93, 1993. Available via anonymous ftp from rutcor.rutgers.edu, file /pub/rrr/reports93/27.ps.gz.

2. T. Biedl, G. Kant, A better heuristic for orthogonal graph drawings, Proc. of the 2nd European Symp. on Algorithms (ESA 94), Lecture Notes in Comp. Science 855, Springer-Verlag (1994), pp. 124-135.

3. T. Biedl, New Lower Bounds for Orthogonal Graph Drawings, Rutcor Research Report 19-95, 1995. Available via anonymous ftp from rutcor.rutgers.edu, file $/$ pub/rrr/reports95/19.ps.gz.

4. T. Biedl, Orthogonal Graph Drawings: Algorithms and Lower Bounds, Diploma thesis TU Berlin (to appear).

5. M. Formann, F. Wagner, The VLSI layout problem in various embedding models, Graph-Theoretic Concepts in Comp. Science (16th Workshop WG'g0), SpringerVerlag, Berlin/Heidelberg, 1992, pp. 130-139.

6. A. Garg, R. Tamassia, On the computational complexity of upward and rectilinear planarity testing, Proc. Graph Drawing '94, Lecture Notes in Comp. Science 894, Springer Verlag (1994), pp. 286-297

7. G. Kant, Drawing planar graphs using the lmc-ordering, Proc. 33th Ann. IEEE Symp. on Found. of Comp. Science 1992, pp. 101-110, extended and revised version to appear in Algorithmica, special issue on Graph Drawing.

8. M.R. Kramer, J, van Leeuwen, The complexity of wire routing and finding minimum area layouts for arbitrary VLSI circuits. Advances in Computer Research, Vol. 2: VLSI Theory, JAI Press, Reading, MA, 1992, pp. 129-146.

9. F.T. Leighton, New lower bounds techniques for VLSI, Proc. 22nd Ann. IEEE Symp. on Found. of Comp. Science 1981, pp. 1-12.

10. P. Rosenstiehl, R.E. Tarjan, Rectilinear planar layouts and bipolar orientations of planar graphs, Discr. and Comp. Geometry 1 (1986), pp. 343-353.

11. J.A. Storer, On minimal node-cost planar embeddings, Networks 14 (1984), pp. 181-212.

12. R. Tamassia, I.G. Tollis, A Unified Approach to Visibility Representations of Planar Graphs, Disc. Comp. Geom. 1 (1986), pp. 321-341.

13. R. Tamassia, I.G. Tollis, Efficient embedding of planar graphs in linear time, Proc. IEEE Int. Symp. on Circuits and Systems (1987), pp. 495-498.

14. R. Tamassia, I.G. Tollis, Planar grid embedding in linear time, IEEE Trans. Circ. Syst. 36 (9), 1989, pp. 1230-1234.

15. R. Tamassia, I.G. Tollis, J.S. Vitter, Lower bounds for planar orthogonal drawings of graphs, Inf. Proc. Letters 39 (1991), pp. 35-40.

16. L.G. Valiant, Universality considerations in VLSI circuits, IEEE Trans. on Comp. C-30 (2), 1981, pp. 135-140. 